\title{
Local Quantum Critical Point and Non-Fermi Liquid Properties
}

\author{
Qimiao Si \\ Department of Physics \& Astronomy, Rice University, Houston,TX 77005-1892, \\ U.S.A.
}

\begin{abstract}
Quantum criticality provides a means to understand the apparent nonFermi liquid phenomena in correlated electron systems. How to properly describe quantum critical points in electronic systems has however been poorly understood. The issues have become particularly well-defined due to recent experiments in heavy fermion metals, in which quantum critical points have been explicitly identified. In this paper, I summarize some recent theoretical work on the subject, with an emphasis on the notion of "local quantum criticality". I describe the microscopic work based on an extended dynamical mean field theory, as well as Ginzburg-Landau arguments for the robustness of the local quantum critical point beyond the microscopics. I also present the consequences of this picture on the inelastic neutron scattering, NMR, Fermi surface properties and Hall coefficient, and compare them with the available experiments. Some analogies with the Mott transition phenomena are also noted.
\end{abstract}

\section{Introduction}

One basic issue in correlated electron systems concerns how electron-electron interactions lead to non-Fermi liquid behavior. Proximity to a quantum critical point (QCP) provides one mechanism. Fermi liquid theory for spatial dimensions higher than one is internally consistent when the electron-electron interactions are treated perturbatively. At a QCP, however, the effective interactions can become very strong due to quantum critical fluctuations, opening the door to a non-Fermi liquid critical state.

Quantum critical metals are of general interest in a variety of strongly correlated electron systems, possibly also for high temperature superconductors[1]. The issues are however particularly well-defined in heavy fermion metals, for the simple reason that QCPs have been explicitly identified. Here, the transitions are typically between a paramagnetic metal and an antiferromagnetic metal. For instance, $\mathrm{CeCu}_{6-\mathrm{y}} \mathrm{Au}_{\mathrm{y}}$ becomes magnetic when the Au-doping reaches $y_{c} \approx 0.1\left[2\right.$, the stoichiometric $\mathrm{YbRh}_{2} \mathrm{Si}_{2}$ is fortuitously sited $\left(T_{N} \approx 70 \mathrm{mK}\right)$ close to its QCP [3], and in the cases of $\mathrm{CePd}_{2} \mathrm{Si}_{2}$ and $\mathrm{CeIn}_{3}$ the Néel temperature can be suppressed by applying pressure[4. In the quantum critical regime, these materials indeed show non-Fermi liquid properties. The electrical resistivity is linear (or close to being linear) in $T$, for as extended a temperature range 
as three decades [5]. The specific heat coefficient is either singular - so that the effective mass diverges in the $T=0$ limit - or is finite but contains a non-analytic dependence on temperature. There is no doubt that such non-Fermi liquid behavior originates from quantum critical physics, as Fermi liquid properties (constant specific heat coefficient and/or $T^{2}$ resistivity) are recovered at low temperatures when the system is tuned away from the QCP [6, 2, 7, 8].

Some direct clues to the nature of such metallic QCPs have come from the inelastic neutron scattering experiments of Schröder et al. 9, 10]. The frequency and temperature dependences of the dynamical spin susceptibility are characterized by an anomalous exponent $\alpha<1$ as well as $\omega / T$ scaling. In addition, the same anomalous exponent $\alpha$ is seen essentially everywhere in the Brillouin zone. These experimental results differ in a very basic fashion from the standard Hertz-Millis picture, which argues for a Gaussian fixed point 11]. The Gaussian picture is formulated entirely in terms of paramagnons the long-wavelength fluctuations of the magnetic order parameter. The critical theory is the $\phi^{4}$ theory, describing the non-linear couplings of the paramagnons. Other degrees of freedom, including fermions, are considered to be bystanders; the primary effect of fermions is to cause a Landau damping, making the dynamic exponent, $z$, larger than 1. In the antiferromagnetic case, the damping is linear in frequency and $z=2$. For either three or two spatial dimensions $(d)$ the effective dimension, $d_{\text {eff }}=d+z$, is larger than or equal to 4 , the upper critical dimension of the $\phi^{4}$ theory. The fixed point is therefore Gaussian. As a result, the dynamical spin susceptibility would have to have the mean-field form,

$$
\chi^{\text {Gaussian }}(\mathbf{q}, \omega) \sim \frac{1}{(\mathbf{q}-\mathbf{Q})^{2}-i \omega},
$$

where $\mathbf{Q}$ is the antiferromagnetic ordering wavevector.

The search for non-Gaussian quantum critical metals has proceeded along a number of directions [12, 13, 14, 15]. Here, I describe in some detail the work on the local quantum critical point 12, 16, 17, 18, which can confront the existing experiments.

\section{Model and microscopic approach}

We focus on the Kondo lattice Hamiltonian,

$$
H=\sum_{i j, \sigma} t_{i j} c_{i \sigma}^{\dagger} c_{j \sigma}+\sum_{i} J_{K} \mathbf{S}_{i} \cdot \mathbf{s}_{c, i}+\frac{1}{2} \sum_{i j} I_{i j} \mathbf{S}_{i} \cdot \mathbf{S}_{j} .
$$

A lattice of spin- $\frac{1}{2}$ local moments $\left(\mathbf{S}_{i}\right)$ and a conduction electron band $\left(c_{i \sigma}\right)$ are coupled through an antiferromagnetic Kondo exchange interaction $\left(J_{K}\right)$ and an RKKY interaction $\left(I_{i j}\right)$. The number of conduction electrons per unit cell, $x$, is taken to be close to but (without loss of generality) less than 1; all the phases are metallic. Two limits of this model are well-understood [19, 20]. When the RKKY interaction is negligible, the local moments are expected to be completely screened by the spins of the conduction electrons. The resulting Kondo resonances turn the local moments into a part of the 


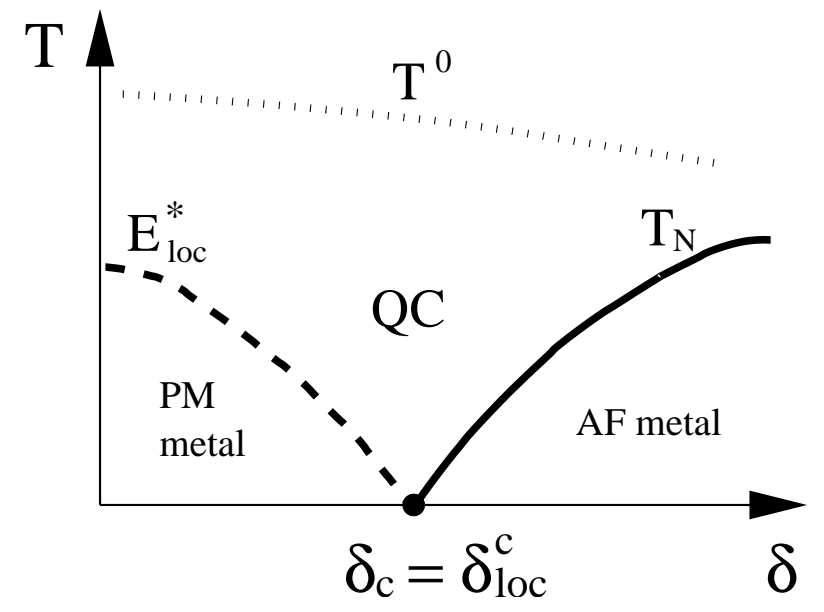

Figure 1. Local quantum critical point. The RKKY interaction $I \equiv T_{K}^{0} \delta$ is tuned as the bare Kondo scale $T_{K}^{0}$ is kept fixed. The local susceptibility is Pauli below $E_{l o c}^{*}$, Curie above $T^{0}$, and logarithmic [cf. Eq. (5)] in the quantum critical (QC) regime.

electronic excitations below some energy scale $E_{l o c}^{*}$ (Fig. 1). In the opposite limit, the dominating RKKY interactions lead to an antiferromagnetic metal.

To treat the dynamical competition between the RKKY and Kondo interactions, we have applied an extended dynamical mean field theory (EDMFT) 21, 22]. The key quantities are the usual electron self-energy, $\Sigma(\omega)$, and a "spin self-energy", $M(\omega)$, which enters the dynamical spin susceptibility as follows [21]

$$
\chi(\mathbf{q}, \omega)=\frac{1}{I_{\mathbf{q}}+M(\omega)},
$$

where $I_{\mathbf{q}}$ is the spatial Fourier transform of $I_{i j}$. The key advantage of the approach is that here spin-damping is no longer assumed to be due to a decay into quasiparticlequasihole pairs. [Such an assumption is inherent in the paramagnon approach, and is responsible for a linear $\omega$-dependence in $M(\omega)$ - cf. Eq. (11).] The approach sets out to also determine whether the fermionic excitations at the QCP retain the heavyquasiparticle character. Within EDMFT, the lattice model is studied through a selfconsistent impurity model, the Bose-Fermi Kondo model:

$$
\begin{aligned}
H_{\mathrm{imp}}= & J_{K} \mathbf{S} \cdot \mathbf{s}_{c}+\sum_{p, \sigma} E_{p} c_{p \sigma}^{\dagger} c_{p \sigma} \\
& +g \mathbf{S} \cdot \sum_{p}\left(\phi_{p}+\phi_{-p}^{\dagger}\right)+\sum_{p} w_{p} \phi_{p}^{\dagger} \cdot \phi_{p},
\end{aligned}
$$

where $g, E_{p}$ and $w_{p}$ are determined self-consistently [12], and $\phi_{p}$ describes a fluctuating magnetic field.

\section{Local quantum critical point}

We find two types of QCP 12]. The more exotic type is the LQCP, as illustrated in Fig. 1. Here, the local energy scale, $E_{l o c}^{*}$, vanishes at the QCP. The local Kondo physics 
is itself critical, and this criticality is embedded in the criticality (i.e. an infinite spatial correlation length) associated with the magnetic ordering transition.

Corresponding to the vanishing $E_{l o c}^{*}$ is a divergent local (i.e., the $\mathbf{q}$-averaged) susceptibility. The specific form of the divergence is logarithmic,

$$
\chi_{l o c}(\omega)=\frac{1}{2 \Lambda} \ln \frac{\Lambda}{-i \omega},
$$

where $\Lambda \approx T_{K}^{0}$. The spin self-energy is

$$
M(\omega) \approx-I_{\mathbf{Q}}+A(-i \omega)^{\alpha} .
$$

Here, $I_{\mathbf{Q}}$ is the value of $I_{\mathbf{q}}$ at the ordering wavevector $\mathbf{q}=\mathbf{Q}$, and the exponent is given by

$$
\alpha=\frac{1}{2 \rho_{I}\left(I_{\mathbf{Q}}\right) \Lambda},
$$

where $\rho_{I}\left(I_{\mathbf{Q}}\right)$ is the "RKKY density of states", $\rho_{I}(\epsilon) \equiv \sum_{\mathbf{q}} \delta\left(\epsilon-I_{\mathbf{q}}\right)$ at $\epsilon=I_{\mathbf{Q}}$.

Viewed from the effective local Kondo model Eq. (4), the vanishing $E_{l o c}^{*}$ corresponds to placing this local problem on its critical manifold (a separatrix in the $J_{K}-g$ parameter space). Indeed, the LQCP was first found[12] using an $\epsilon \equiv 1-\gamma$ expansion [23, 24] for Eq. (44), along with the self-consistency conditions. Here $\gamma$ is the exponent that characterizes the spectrum of the fluctuating magnetic field: $\sum_{p}\left[\delta\left(\omega-w_{p}\right)-\delta\left(\omega+w_{p}\right)\right] \sim|\omega|^{\gamma} \operatorname{sgn} \omega$. The effects of spin-anisotropy (XY and Ising) have been treated in a similar fashion[17, 18. More recently, numerical studies have been carried out[16] for the EDMFT equations in the anisotropic Kondo lattice model, using a Quantum Monte Carlo algorithm originally developed by Grempel and Rozenberg[25]. The numerical results are consistent with the logarithmic form for the singular local susceptibility at the LQCP [cf. Eq. (5)]. The numerical value for the exponent $\alpha$ is about 0.7 , close to what is seen experimentally in $\mathrm{CeCu}_{6-\mathrm{y}} \mathrm{Au}_{\mathrm{y}}$.

Since the peak susceptibility, $\chi(\mathbf{Q}, \omega)$, is divergent at the QCP, the basic condition for realizing a LQCP is such that the $\mathbf{q}$-averaged susceptibility diverges at the same point where the peak susceptibility does. Two dimensional magnetic fluctuations satisfy this condition. If the magnetic fluctuations are purely three dimensional, and if there is no frustration, then $E_{l o c}^{*}$ would be finite at the QCP corresponding to a crossover scale towards the eventual Gaussian behavior [12, 26]. We also note that our analysis applies provided the $T=0$ transition is continuous. The latter can be explicitly checked by studying the EDMFT equations on the ordered side (in the presence of a self-consistent static field); work along this direction is in progress.

\section{Robustness of the LQCP}

Based on Ginzburg-Landau considerations [12, we have also argued that the LQCP is robust beyond the microscopic EDMFT provided $\alpha<1$. The key issue is whether the local susceptibility at the QCP remains divergent when we allow for a $\mathbf{q}$-dependence 
in the self-energies. Writing the general scaling form for the static spin susceptibility for $\mathbf{q}$ in the vicinity of the ordering wavevector $\mathbf{Q}$,

$$
\chi(\mathbf{q}) \sim \frac{1}{(\mathbf{q}-\mathbf{Q})^{2-\eta}},
$$

the question becomes equivalent to whether the spatial anomalous dimension $\eta$ remains equal to zero: if it is, then the corresponding local susceptibility in two dimensions remains singular. For $\alpha<1$, the non-linear couplings among the long-wavelength modes are irrelevant; their contributions to the spin self-energy will contain a $\mathbf{q}$-dependence that is at most $(\mathbf{q}-\mathbf{Q})^{2}$. This, coupled with the fact that the contributions to the spin self-energy from the local modes are expected to be smooth in $\mathbf{q}$, lead to the conclusion that $\eta=0$. The LQCP is therefore internally consistent.

To reiterate, the tuning to the $\mathrm{QC}$ regime provides a robust mechanism for a singular local susceptibility, which in turn places the local (Kondo) fluctuations exactly at its own criticality.

\section{Quantum-critical dynamics, Fermi surface properties, and experiments}

We now turn to experimentally testable properties:

- The dynamical spin susceptibility is

$$
\chi(\mathbf{q}, \omega)=\frac{1}{\left(I_{\mathbf{q}}-I_{\mathbf{Q}}\right)+A(-i \omega)^{\alpha} W(\omega / T)} .
$$

- The static uniform spin susceptibility has a modified Curie-Weiss form,

$$
\chi(T)=\frac{1}{\Theta+B T^{\alpha}},
$$

with exactly the same exponent as in Eq. (9).

- The NMR relaxation rate contains a temperature-independent component:

$$
\frac{1}{T_{1}} \sim A_{h f}^{2} \frac{\pi}{8 \Lambda}
$$

- The Fermi surface changes sharply at the QCP, from "large" [volume $(1+x)$ ] to "small" (volume $x$ and a different topology) as the system orders.

Eq. (9) reproduces the form observed [9] in the inelastic neutron scattering experiments on $\mathrm{CeCu}_{6-\mathrm{y}} \mathrm{Au}_{\mathrm{y}}$. In addition, the neutron results are consistent with twodimensional magnetic fluctuations [10].

Recently, NMR experiments have been carried out [27] in $\mathrm{YbRh}_{2} \mathrm{Si}_{2}$. The relaxation rate $\frac{1}{T_{1}}$ is strongly dependent on the magnetic field, in a way that appears consistent with $B / T$ scaling. Over the temperature range where the specific heat is logarithmic, $\left(\frac{1}{T_{1}}\right)_{B \rightarrow 0}$ does contain a constant component.

Even more recently, NQR experiments have been carried out 28 at a $\mathrm{Cu}$ site in $\mathrm{CeCu}_{6-\mathrm{y}} \mathrm{Au}_{\mathrm{y}}$. The relaxation rate has a non-Korringa temperature dependence; at low temperatures $\frac{1}{T_{1}} \sim T^{\beta}$, where the exponent $\beta \approx 0.75$ is very close to the fractional 
exponent $\alpha$ seen in the $\mathbf{q}$-dependent dynamical spin susceptibility [9]. This result would be consistent with the neutron scattering result if the hyperfine couplings between the $\mathrm{Cu}$ nuclei and the $f$-electron spins at the Ce sites are such that the dominant contributions to $\frac{1}{T_{1}}$ come from generic wavevectors (wavevectors far away from the peak wavevectors). With this assumption about the hyperfine coupling, the Cu-site NQR experiment confirms the existence of the fractional exponent over an extended region of the Brillouin zone.

In addition to $\mathrm{CeCu}_{6-\mathrm{y}} \mathrm{Au}_{\mathrm{y}}$, the modified Curie-Weiss form for the uniform spin susceptibility, Eq. (10), has also been seen [5] in $\mathrm{YbRh}_{2} \mathrm{Si}_{2}$.

The Fermi surface reconstruction can be probed through de Haas-van Alphen experiments. We are aware of one case, $\mathrm{CeRh}_{2} \mathrm{Si}_{2}$ (the quantum-critical behavior of which is not yet as well characterized as for the other heavy fermions), in which such measurements do reveal a Fermi-surface reconstruction [29]. A less direct experimental signature is that the Hall coefficient should jump as the system is tuned through the QCP [30, 12]; some preliminary indication for such a behavior has been found [31] in $\mathrm{YbRh}_{2} \mathrm{Si}_{2}$.

We make two remarks in passing. First, similar features in the dynamics $\left(\omega / T\right.$-scaling) [32, 33] and other properties [34, 35] occur in $\mathrm{UCu}_{5-x} \mathrm{Pd}_{x}$, raising the possibility that local criticality is also operating in these (strongly disordered and frustrated) systems. Second, we have focused on the quantum-critical physics associated with a transition between paramagnetic and antiferromagnetic metals. Other phases, such as spin-liquids [36, 37, may also be relevant [14].

\section{Concluding remarks}

We have identified a local quantum critical metal, which is described by a nonGaussian fixed point. This picture provides a natural explanation for some most unusual experiments in heavy fermion metals in the vicinity of a magnetic quantum critical point.

The local quantum critical point serves as a concrete example in which fermions, instead of being bystanders, directly participate in the critical behavior. The Kondo resonances, which have the quantum numbers of an electron and would turn the local moments into a part of the electron fluid, become critical at the same point where a magnetic ordering sets in.

More generally, the local moment physics in Kondo systems is analogous to the Mott phenomenon. The strong Coulomb interactions lead to a microscopic Coulombblockade or, equivalently, a projection onto a restricted Hilbert space. Such effects are assumed to be inconsequential in the Gaussian quantum critical metal picture. On the other hand, and reminiscent of what happens in the usual Mott transition, they play a central role in a local quantum critical metal.

I would like to thank D. Grempel, K. Ingersent, S. Rabello, J. L. Smith, and L. Zhu for collaborations, many colleagues - particularly P. Coleman, J. Custers, J. Flouquet, K. Ishida, H. v. Löhneysen, G. Lonzarich, Y. Ōnuki, S. Paschen, C. Pépin, A. Rosch, 
S. Sachdev, F. Steglich, and R. Walstedt - for discussions, and NSF Grant No. DMR0090071, TcSAM, and the Robert A. Welch foundation for support.

\section{References}

[1] Panagopoulos C et al., Solid State Commun. 126, 47 (2003) and references therein;

Tallon J L et al., Phys. Stat. Sol. (b) 215, 531 (1999).

[2] Löhneysen H v et al., Phys. Rev. Lett. 72, 3262 (1994);

Löhneysen H v, J. Phys. Cond. Matt. 8, 9689 (1996).

[3] Trovarelli O et al., Phys. Rev. Lett. 85, 626 (2000).

[4] Mathur N D et al., Nature 394, 39 (1998).

[5] Steglich F et al., proceedings of LT23.

[6] Gegenwart P et al., Phys. Rev. Lett. 89, 056402 (2002).

[7] Grosche F M et al., J. Phys. Cond. Matt. 13, 2845 (2001).

[8] Demuer A et al., J. Phys. Cond. Matt. 13, 9335 (2001);

Knebel G et al., Phys. Rev. B 65, 024425 (2002).

[9] Schröder A et al., Nature 407, 351 (2000).

[10] Stockert O et al., Phys. Rev. Lett. 80, 5627 (1998).

[11] For a review, see Sachdev S, Quantum Phase Transitions (Cambridge Univ. Press, Cambridge, 1999), chap. 12.

[12] Si Q, Rabello S, Ingersent K, and Smith J L, Nature 413, 804 (2001);

cond-mat/0202414

[13] Coleman P, Pépin C, and Tsvelik A M, Phys. Rev. B 62, 3852 (2000).

[14] Sachdev S and Morinari T, Phys. Rev. B 66, 235117 (2002).

[15] Abanov A and Chubukov A, Phys. Rev. Lett. 84, 5608 (2000).

[16] Grempel D and Si Q, cond-mat/0207493.

[17] Zhu L and Si Q, Phys. Rev. B 66, 024426 (2002).

[18] Zaránd G and Demler E, Phys. Rev. B 66, 024427 (2002).

[19] Doniach S, Physica B 91, 231 (1977).

[20] Varma C M, Rev. Mod. Phys. 48, 219 (1976).

[21] Si Q and Smith J L, Phys. Rev. Lett. 77, 3391 (1996);

Smith J L and Si Q, Phys. Rev. B 61, 5184 (2000).

[22] Chitra R and Kotliar G, Phys. Rev. Lett. 84, 3678 (2000).

[23] Smith J L and Si Q, Europhys. Lett. 45, 228 (1999).

[24] Sengupta A M, Phys. Rev. B 61, 4041 (2000).

[25] Grempel D and Rozenberg M, Phys. Rev. B 60, 4702 (1999).

[26] Burdin S, Grempel D R, and Georges A, Phys. Rev. B 66, 045111 (2002).

[27] Ishida K et al., Phys. Rev. Lett. 89, 107202 (2002); preprint (2003).

[28] Walstedt R E, Kojima H, Butch N, and Bernhoeft N, Phys. Rev. Lett. 90, 067601 (2003).

[29] Araki S et al., Phys. Rev. B 64, 224417 (2001).

[30] Coleman P, Pépin C, Si Q, and Ramazashvili R, J. Phys. Cond. Matt. 13, R723 (2001).

[31] Paschen S et al., Acta Phys. Pol. B34, 359 (2003).

[32] Aronson M C et al., Phys. Rev. Lett. 75, 725 (1995).

[33] MacLaughlin D E et al., Phys. Rev. Lett. 87, 066402 (2001).

[34] Maple M B et al., J. Low Temp. Phys. 95, 225 (1994).

[35] Stewart G R, Rev. Mod. Phys. 73, 797 (2001).

[36] Sachdev S and Ye J, Phys. Rev. Lett. 70, 3339 (1993).

[37] Parcollet O and Georges A, Phys. Rev. B 59, 5341 (1999). 